\title{
Adaptive Fitting Reference Frame for 2-D Indoor Localization Based on RFID
}

\author{
Zhixin Yang and Chen Lei
}

\begin{abstract}
Indoor localization information is important to many different industries. Ever-rising demand from both custom and supplier pose great challenge for indoor localization technology. The emergence of rapid development of Radio Frequency Identification (RFID) technology enriches indoor positioning methodology. Traditional indoor localization algorithm mainly based on the signal propagation property. Among all of positioning algorithm, LANDMARC is most promising one but still has some limitation such as multipath effect, noise form environment and quantity constrain of tags. This paper identifies previous RFID-based indoor localization algorithm and proposes a new algorithm aiming at delivering above limitation to estimate objective position indoor environment. It use RSS propagation curve to obtain RSS value of virtual reference tag, and then estimate target tags position. To evaluate its performance, a comparison between LANDMARC and proposed algorithm was conducted. Experiment result show proposed algorithm is more accurate than LANDMARC.
\end{abstract}

Index Terms-RFID, indoor localization, LANDMARC, rss curve.

\section{INTRODUCTION}

Indoor location has attracted a lot of attention in the past decades, both in academic research and industrial application Several indoor localization methods have been developed and discussed. But different kinds of limitation constrain its application. RFID (Radio Frequency Identification), as an emerging technology, has been considered as the most popular device in positioning application because of its unique character such as non-contact, low-cost, durable and huge data capability. [1] give a brief introduction of this kind of new technology. RFID is ideal tool of capturing data because of its unique character and it has been widely used in many fields, including manufacturing industry management, supply chain and many other public organizations. [2] present a systematic describe in different industrial application.

Based on the propagation of signal, there are several algorithms to estimate object position nowadays. During the propagation process, the strength of signal decreases along with distance increase. But the signal strength distribution model is complex due to interference from different propagation environment. Some author utilized Received Signal Strength Indication (RSSI) to estimate the distance between reference tags and tracking tag. [3] has divided the RSSI propagation curve into four phase due to RSSI loss

Manuscript received April 22, 2013; revised July 15, 2013.

The authors are with the Department Electromechanical Engineering, University of Macau (e-mail: zxyang@umac.mo; MB15550@umac.mo). distribution is nonlinear and then adapted the adjusted function to estimate distance. Besides, many other methods were applied to estimate the position. Time-of arrival (TOA) is a kind of distance estimation method through calculating the signal propagation distance. Similar method like time difference of arrival (TDOA), the one calculate distance base on time difference. According to other characteristic of signal, phase of arrival (POA) and angle of arrival (AOA) have been studied for years. By analysis former academic research work and experiment, [4] develop a totally different positioning method called LANDMARC which defined several reference tags first time. By utilization received signal strength indicator (RSSI) and K-nearest neighbour (KNN) algorithm was adopted to calculate the position of tracking tags. This method utilized reference tags' position (known) to estimated tracking tag's position (unknown). The accuracy of LANDMARC positioning algorithm relies on the quantity of reference tags. In some extent, more reference tags will increase positioning accuracy. But excessive reference tags also will increase cost and bring unexpected signal interference as result in decreasing the positioning accuracy.[5] has proposed an algorithm called Prior Measurement Error-correcting (PME) to balance this problem. It use positioning error of several specific points which near to tracking point to calibrate tracking tag coordinate calculated by LANDMARC algorithm. [6] has contributed an idea to improve the positioning accuracy by means of eliminating diversity of tags. [7] has proposed a method to overcome the defect of LANDMARC algorithm caused by high density of reference tags and high complexity of computing.[8] introduce event-based possibility model to describe experiment environment interference and use PSO-based optimal algorithm to optimize positioning result. Though RFID localization offers impressive benefits, it still has some defect to overcome. [9] is employed adaptive $\mathrm{k}$ value to enhance positioning accuracy.[10] gives an comprehensive analysis of accuracy-influence factors. This paper presents the state-of-art technology of RFID location algorithm and some improvements. In the following of this paper, we will introduce the RFID system in section two and LANDMARC localization algorithm in section three. New algorithm will propose in section four. Finally, the calibration of new algorithm and experiment will present in section five and six.

\section{The Proposed Algorithm}

[11] introduced a new concept VIRE (virtual reference tag) and the key idea of this algorithm is to eliminate some impossible location without utilization more real tags. The 
author divided virtual grid cell which covered by four real reference tags into finer virtual grid cells and each grid cell can be regard as covered by four virtual reference tag. The RSSI value of those virtual reference tags calculate by linearly interpolation algorithm. However, the relationship between signal strength and distance indoor cannot easily regard as linear. When the distance between reader and tag is small, the signal strength change repaid with distance which means a small distance increase or decrease will lead to huge variation of signal strength. When the distance between reader and tags is large, the signal strength is not as sensitive as before. The estimated RSSI of virtual tags cannot reach a high accuracy as result in the target tags having low accuracy. The RSSI value of virtual reference tags rely on real reference tags. Not only the density of reference tags has influence the estimation error, but also many other factors, such as indoor furniture layout, construction structure, people movement. Moreover, the uncertainty of factors influence will also limit the improvement effort. If some real reference tags RSS value has fluctuation or contain noise, it will pass even magnify the influence to virtual reference tags

It is necessary and vital for us to know about the curve of signal strength propagation under experiment environment. For this purpose, tags will be fixed at different distance to reader to collect the signal data. Fig. 1 shows that tag has been put at different distance along straight line. Three kinds of interval $10 \mathrm{~cm}, 15 \mathrm{~cm}$ or $20 \mathrm{~cm}$ were be chose to capture signal data.

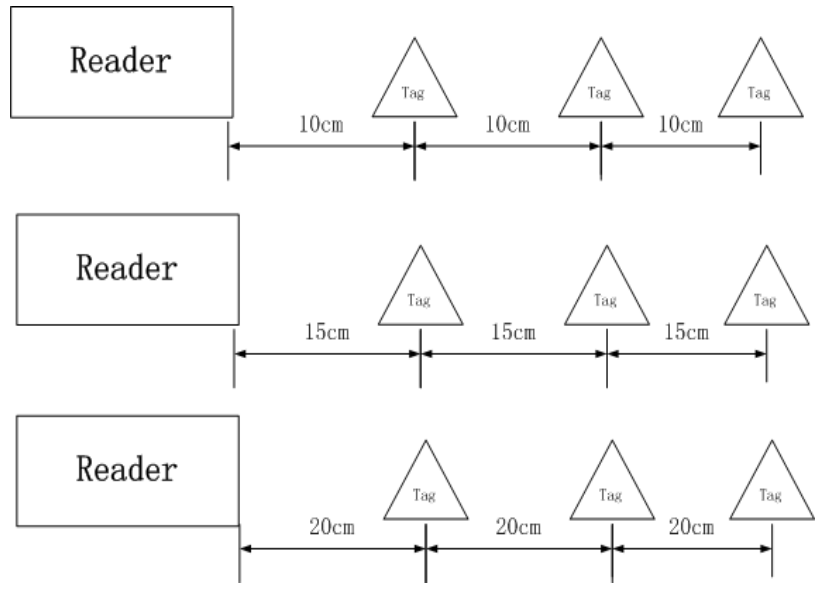

Fig. 1. Indoor RSS measurement

According to captured RSS value, the signal strength propagation curve can obtain. We fix RFID reader firstly and put active tag at every $10 \mathrm{~cm}, 15 \mathrm{~cm}, 20 \mathrm{~cm}$ away from reader and measurement range is $420 \mathrm{~cm}$. Fig. 2 depicts the propagation curve, the blue points represents the data measured in experiment. Signal is sensitive to distance when measurement range is small than $100 \mathrm{~cm}$ and large than $300 \mathrm{~cm}$. From $100 \mathrm{~cm}$ to $300 \mathrm{~cm}$, the RSS value almost same despite of variety of distance. After excluding some extreme data which may effects by occasional factors, those data can be fitted at different order polynomial function. The discreet value fitted at $3^{\text {th }}, 4^{\text {th }}, 5^{\text {th }}$ degree polynomial model by MATLAB. Their RMSE are 4.793, 4.735, 4.841, receptively. It is obvious that $4^{\text {th }}$ degree polynomial function can describe this propagation better.

$$
f(x)=p_{1} x^{4}+p_{2} x^{3}+p_{3} x^{2}+p_{4} x+p_{5}
$$

where $p_{1}=8.655 \mathrm{e}-009, \quad p_{2}=-9.791 \mathrm{e}-006, \quad p_{3}=0.003669$, $p_{4}=-0.6012, p_{5}=-35.68$

As analysis before, the reference tags have a great influence on estimation accuracy. The more reference tags will increase accuracy of tags in some extent. But the paradox is that more reference tags will cause severe multi-path effect. For solve this issue, the idea of virtual tags has been introduced to positioning algorithm. The real reference tags distributed evenly indoor environment and form a two-dimensional grid. Each node means unique reference tag with known coordinates and RSS value captured by reader. Each cell covered by 4 real reference tags will divide into finer grid and each knot refer to a virtual reference tag.[11] present the virtual reference tags coordinate concept and gives more detail RSS value of virtual tags. In this paper, the value of virtual tags will calculate by polynomial function (1).

The virtual reference tags can solve constrain caused by quantity of reference tags in some extent. It will improve estimation accuracy as means of increasing the density of reference tags without introduce more real reference tags. Fig. 3 has illustrated the distribution of real reference tags and virtual reference tags.
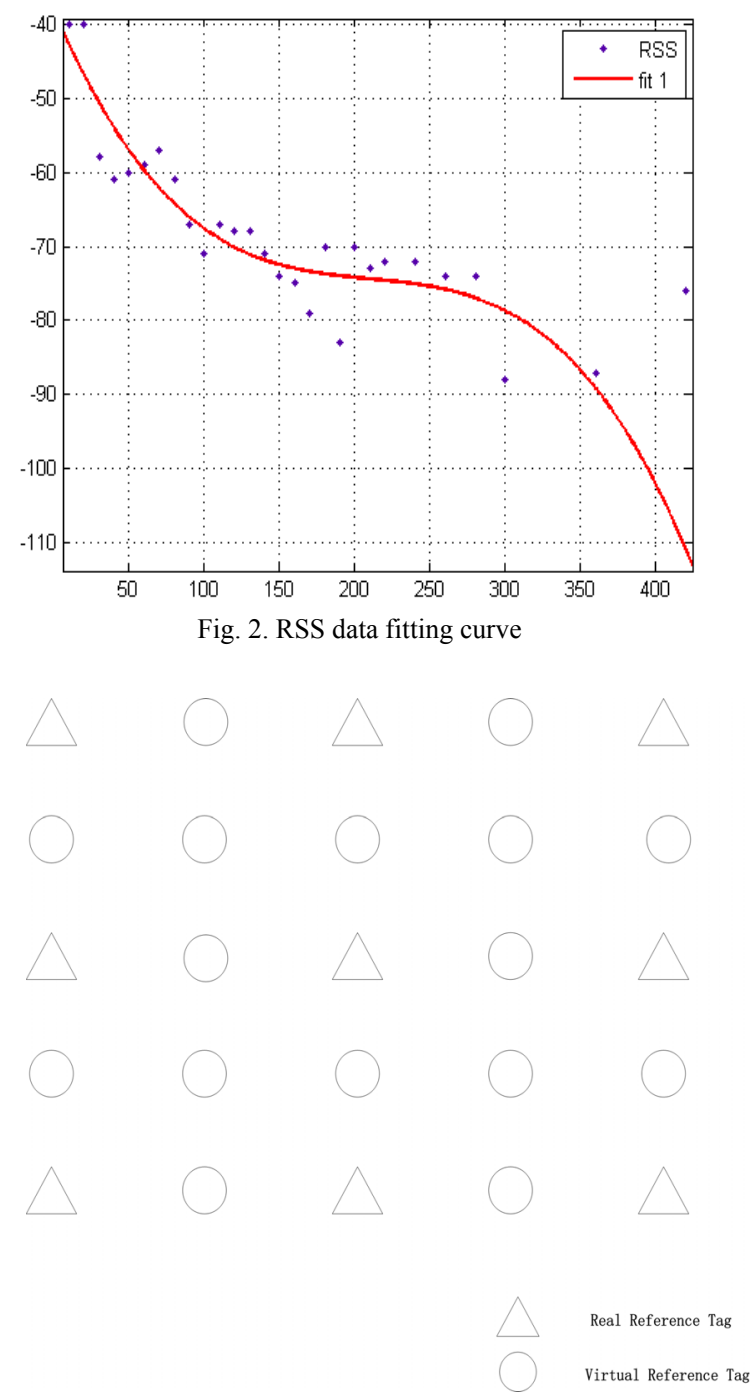

Fig. 3. Arrangement of virtual reference tag 


\section{The CAlibration of Proposed Algorithm}

\section{A. The Reduction of Environment Influence}

For the purpose of further improvement of estimation accuracy, there is essential to deduce other influence from environment and experiment equipment. In some case, even the two tags RSS value obtained by same reader in same position is not equal. [12] has proposed an algorithm to calibrate the error between estimation error on principle of Cross-validation. Firstly, the tracking tag position $(x, y)$ is calculated based on previous algorithm. And then it put $\mathrm{K}$ reference tags and estimated tracking tags into a set

$$
\left\{(x, y),\left(x_{1}, y_{1}\right),\left(x_{2}, y_{2}\right) \ldots \ldots \ldots\left(x_{k}, y_{k}\right)\right\}
$$

By Utilizing the estimated tracking tags position and k-1 reference tags to estimate the $\mathrm{k}$-th reference tag coordinate. Comparing the real reference tags coordinate and estimated one, the difference will be used to calibrate estimated tracking tags position. Repeating this process again and again until the estimated tracking is stable.

$$
\begin{aligned}
& \Delta x=\sum_{i=1}^{k} w_{i}\left(x_{i}^{\prime}-x_{i}\right) \\
& \Delta y=\sum_{i=1}^{k} w_{i}\left(y_{i}^{\prime}-y_{i}\right)
\end{aligned}
$$

where $\left(x_{\mathrm{i}}, y_{\mathrm{i}}\right)$ are the real reference tags coordinate and $\left(x_{i}^{\prime} y_{i}^{\prime}\right)$ are estimated reference tags position.

\section{B. The Elimination of Distribution from Tags}

RFID in door positioning system has utilized many tags as benchmark to provide signal strength so that estimated the tracking tags' position. Ever-rising technique can make RFID tags more complicated and precise which will greatly enhance the accuracy of positioning algorithm, but the error caused by physical structure cannot neglected. It is essential to take some measures to reduce some error by this reason. We introduce the following concept: Basic RSS value (RSSb) and calibrate RSS value (RSSc). RSSb means that reader captures each tag RSS value at a fixed distance. Aiming at improve the accuracy of positioning further, the tag puts every $45^{\circ}$ in sequence at fixed distance to reader and then take the average value of 8 position as RSS b .Fig. 4 demonstrate the distribution of tags and reader. When gets each tag's $\mathrm{RSS}_{\mathrm{b}}$ value, the RSSc can be calculated in the following equation:

$$
R S S_{c}=\frac{\mathrm{RSS}}{\mathrm{RSS}_{b}}
$$

By equation (5), the $R S S I_{c}$ value calculated to represent the RSS which measured value during experiment.

\section{EXPERIMENT AND DISCUSSION}

The experiment conducts in $2 m \times 2 m$ indoor environment including 9 reference tags, which puts every $60 \mathrm{~cm}$. The coordinate of 9 reference are $(0,0),(0,60),(0,120),(60,0)$,
$(60,60),(60,120),(120,0),(120,60),(120,120)$ and 3 target tags are $(90,90),(15,100),(40,50)$. Fig. 5 shows the layout of tags. Three readers have been put in this experiment to collect data.

TABLE I: THE RSS DATA AND COORDINATE OF DIFFERENT TAGS

\begin{tabular}{ccccc}
\hline \hline Tag & Coordinate & Reader 1 & Reader 2 & Reader 3 \\
\hline 1 & $(0,0)$ & -59 & -69 & -71 \\
2 & $(0,60)$ & -68 & -72 & -61 \\
3 & $(0,120)$ & -69 & -72 & -72 \\
4 & $(60,0)$ & -40 & -61 & -72 \\
5 & $(60,60)$ & -65 & -64 & -75 \\
6 & $(60,120)$ & -77 & -68 & -40 \\
7 & $(120,0)$ & -70 & -63 & -74 \\
8 & $(120,60)$ & -67 & -40 & -47 \\
9 & $(120,120)$ & -74 & -67 & -55 \\
Target1 & $(80,80)$ & -67 & -56 & -60 \\
Target2 & $(15,100)$ & -72 & -40 & -70 \\
Target3 & $(40,50)$ & -63 & -67 & -70 \\
\hline
\end{tabular}

Remarks: the three readers has put at three points where can see in Fig. 4.
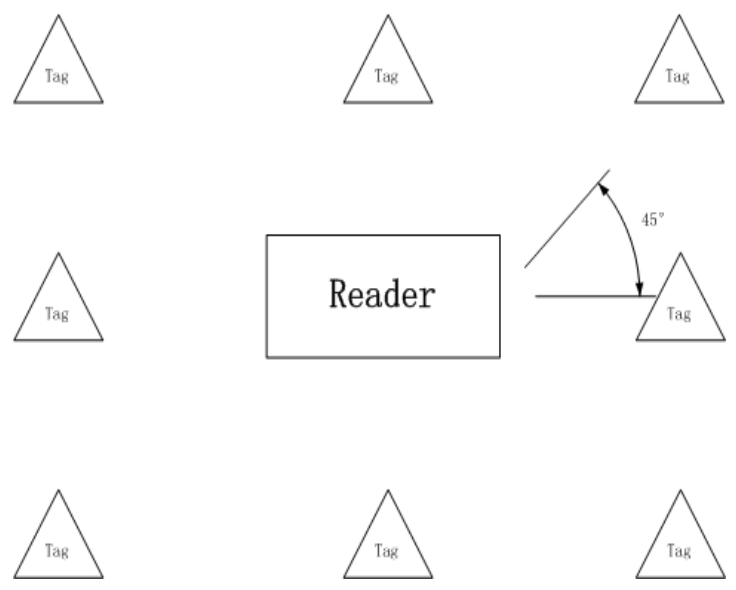

Fig. 4. Elimination arrangement form tags

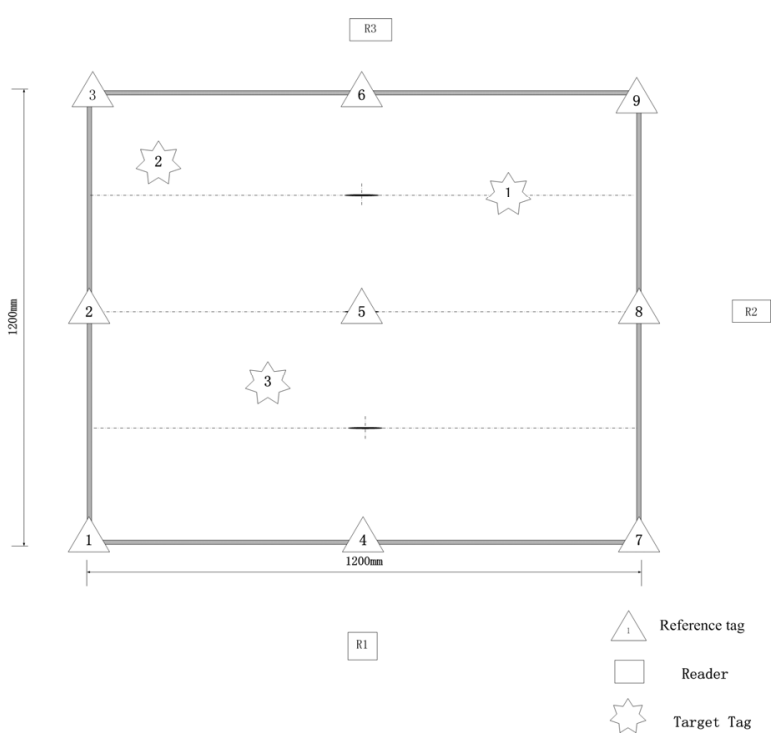

Fig. 5. The layout of real tags, target tags and readers

According to traditional LANDMARC algorithm, the tracking tag position can be computed by above data. The RSS value can be used to analysis relative distance to tracking tag, the similar one has most small distance to target. Different $\mathrm{K}$ has been adapted to computation, k performance 
difference accuracy in this experiment environment. Fig. 6 deploys the relationship between $\mathrm{K}$ and error, less reference tags chosen cannot achieve a high accuracy estimation of tracking tags as result of few position information obtained from known tags. However, more reference tags introduced to estimation still cannot achieve better result because of more disturb brought to computation as well as consideration of cost. When $\mathrm{K}=4$, it has smallest error which means it has most accurate estimation position.

By the measured RSS propagation model indoor environment, virtual reference tags RSS for different readers can obtain. Then, the proposed algorithm can estimate the tracking tag position. Aiming at comparing influence from the number of nearest neighbour reference tags $(K)$, the estimation result based on different number of virtual reference tags has been computed. Fig. 7 demonstrates the relationship between $\mathrm{K}$ and result based on proposed algorithm and the error is lowest when $\mathrm{K}=4$. Similar to LANDMARC algorithm, the relationship between $\mathrm{K}$ and estimation accuracy cannot conclude negative or positive correlation. Table II presents the experiment result.

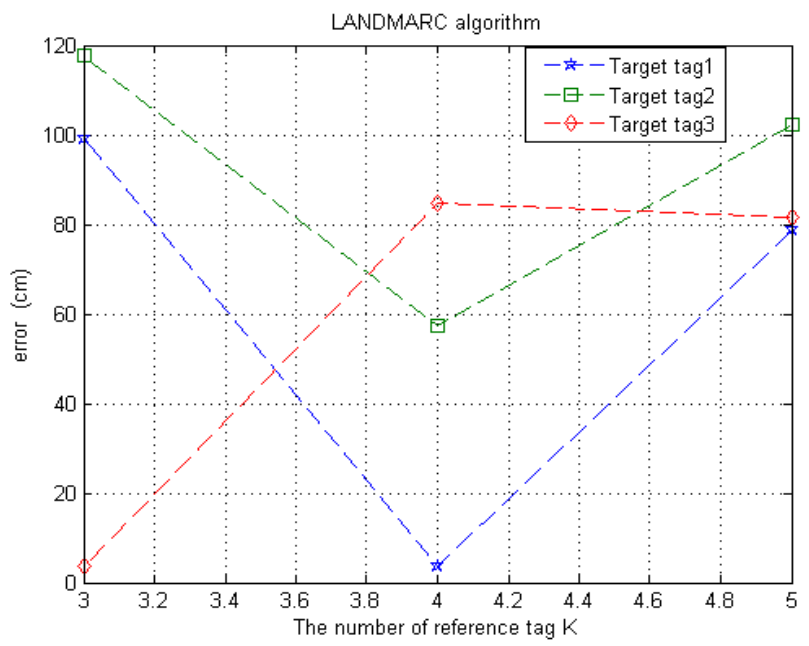

Fig. 6. The localization result of LANDMARC algorithm

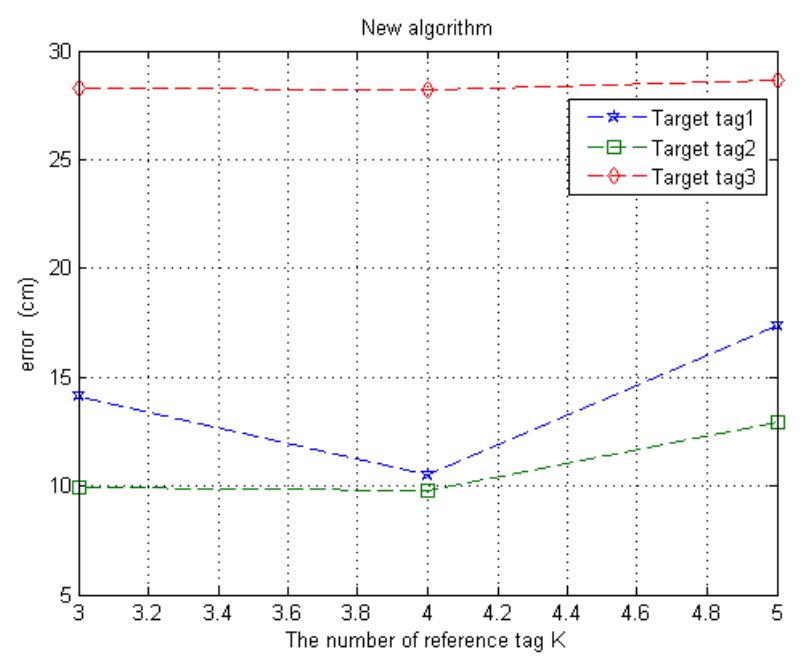

Fig. 7. The localization result of new proposed algorithm

Fig. 8 depicts that new algorithm illustrate better performance with different neighbour reference tags. Accuracy improvement can reach $82 \%$ maximum. In idealist experiment environment, the RSS distribution model can be measured and used to calculate virtual tags which will divide experiment area into smaller region as result of improving accuracy.

TABLE II: THE RESUlt COMPARISON OF TwO ALgORITHM

\begin{tabular}{|c|c|c|c|c|}
\hline \multicolumn{2}{|c|}{ Estimation result } & Target tag 1 & Target tag 2 & Target tag 3 \\
\hline \multirow{3}{*}{ ż } & $\mathrm{K}=3$ & \multirow{6}{*}{$\begin{array}{l}(70.0,80.0) \\
(72.6,77.4) \\
(67.7,72.4) \\
(42.6,49.3) \\
(99.8,57.2) \\
(97.8,58.5)\end{array}$} & \multirow{6}{*}{$\begin{array}{l}(22.0,114.5) \\
(20.7,179.3) \\
(24.2,117.2) \\
(98.2,71.0) \\
(55.7,140.7) \\
(87.4,72.7)\end{array}$} & \multirow{6}{*}{$\begin{array}{l}(20.0,46.7) \\
(20.1,46.6) \\
(19.7,46.8) \\
(42.6,49.4) \\
(99.8,57.2) \\
(97.8,58.5)\end{array}$} \\
\hline & $\mathrm{K}=4$ & & & \\
\hline & $\mathrm{K}=5$ & & & \\
\hline \multirow{3}{*}{ 空至 } & $\mathrm{K}=3$ & & & \\
\hline & $\mathrm{K}=4$ & & & \\
\hline & $\mathrm{K}=5$ & & & \\
\hline
\end{tabular}

New algorithm

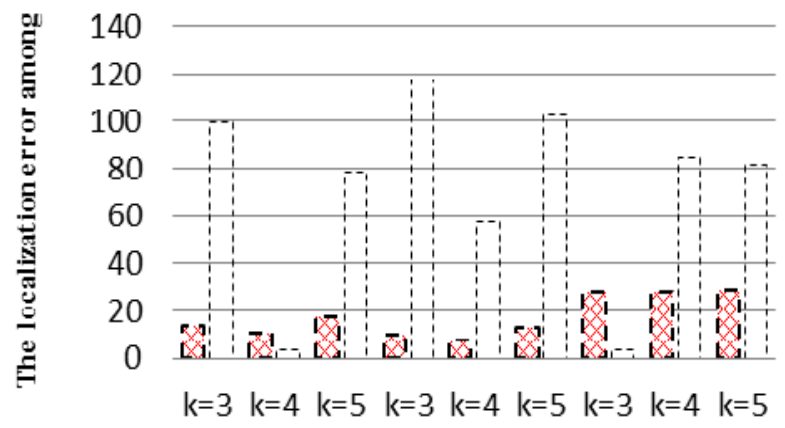

Fig. 8. The localization result comparison between LANDMARC and new algorithm

\section{CONCLUSION}

Indoor localization draws lots of attention from many industries including logistic, traveling and manufacturing. The objective of this paper is to review traditional indoor such as TOA, TDOA, POA localization algorithms based on RFID and provide a hybrid algorithm which mainly based on LANDMARC algorithm and signal propagation model. It can increase estimation accuracy without introduce more reference tags. Indeed, It found the number of nearest neighbour reference tags has great influence on positioning accuracy. Furthermore, the elimination of tag physical difference and environment influence were introduced to calibrate estimation result. Finally the experiment result shows the proposed algorithm has better performance than LANDMARC algorithm which the average accuracy can increase $55 \%$.

\section{REFERENCES}

[1] R. Want, "An introduction to RFID technology," IEEE Pervasive Computing, vol. 5, pp. 25-33, 2006.

[2] X. Zhu et al., "A review of RFID technology and its managerial applications in different industries," Journal of Engineering and Technology Management, vol. 29, pp. 152-167, 2012.

[3] C.-N. Huang, and C.-T. Chan, "ZigBee-based indoor location system by k-nearest neighbor algorithm with weighted RSSI," Procedia Computer Science, vol. 5, pp. 58-65, 2011.

[4] M. N. Lionel et al., "LANDMARC: Indoor location sensing using active RFID," 2004, pp. 701-710.

[5] C. Jing et al., "An efficient algorithm for indoor location based on RFID," in Proc. Wireless Communications and Signal Processing (WCSP), 2011 International Conference, pp. 1-4.

[6] T. Zhang et al., "An improved RFID-based locating algorithm by eliminating diversity of active tags for indoor environment," Computer 
Journal, vol. 52, pp. 902-909, 2009.

[7] J. Guang-yao et al., "An indoor localization mechanism using active RFID tag," in Proc. IEEE International Conference on Sensor Networks, Ubiquitous, and Trustworthy Computing, 2006, pp. 4.

[8] S. Weiguang et al., "Research of optimal placement of active reference tags based on LANDMARC algorithm," in Proc. 2011 6th International Conference on Computer Science \& Education (ICCSE), 2011, pp. 281-285.

[9] H. Kyuwon, and C. Sung Ho, "Advanced LANDMARC with adaptive k-nearest algorithm for RFID location system," in Proc. 2010 2nd IEEE International Conference on Network Infrastructure and Digital Content, 2010, pp. 595-598.

[10] J. Zhou and J. Shi, "A comprehensive multi-factor analysis on RFID localization capability," Advanced Engineering Informatics, vol. 25, pp. 32-40, 2011

[11] Z. Yiyang et al., "VIRE: Active RFID-based localization using virtual reference elimination," in Proc. International Conference on Parallel Processing, 2007, pp. 56-56.

[12] J. Xuejing et al., "An enhanced approach of indoor location sensing using active RFID," in Proc. WASE International Conference on Information Engineering, 2009. ICIE '09., pp. 169-172.

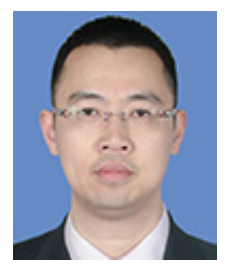

Zhixin Yang was received the B.Eng. in Mechanical Engineering and MPhil (Direct PhD Program) in Intelligent CAD from Huazhong University of Science and Technology, China, and got the Ph.D. in Industrial Engineering and Engineering Management, Hong Kong University of Science and Technology, Hong Kong.

$\mathrm{He}$ is assistant professor in Department Electromechanical Engineering, University of Macau. His interests include Design Informatics, Engineering Management, Intelligent System, Geometric Computation.

Dr. Yang is general secretary, The Association for Promotion of Science \& Technology of Macau and member of American Society of Mechanical Engineers, Institute of Industrial Engineers.

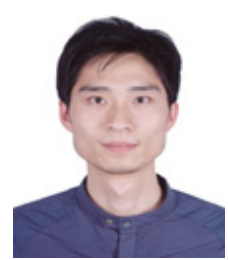

Chen Lei was received B.Eng degree in Electromechanical Engineering from Jilin University, Zhuhai College, China. He is currently working toward the M.Eng degree in Electromechanical Engineering Department, University of Macau.

His research interests include Engineering Management and RFID technology. 